\title{
Modification on the surface of gold nanoparticles with imine formation and cycloaddition reaction
}

\author{
$\underline{\text { Abdolhamid Alizadeh }}^{1 *}$, Changiz Karami ${ }^{2}$, Omid Marvi ${ }^{3}$, Behzad Bahrami $^{1}$, Anita Moradi ${ }^{1}$ and \\ Mohammad Ali Taher ${ }^{4}$
}

\footnotetext{
${ }^{1}$ Department of Organic Chemistry, Faculty of Chemistry, Razi University, Kermanshah 6714967346, Iran.

${ }^{2}$ Department of Chemistry, Kermanshah Branch, Islamic Azad University (IAU), Kermanshah, Iran.

${ }^{3}$ Department of Chemistry, Faculty of Sciences, Payame Noor University (PNU), PO Box 19395-3697, Tehran, Iran.

${ }^{4}$ Department of Chemistry, Faculty of Sciences, Shahid Bahonar University of Kerman, Kerman, Iran.
}

\begin{abstract}
Background: In recent years, the preparation, characterization, and surface modification of nanostructured materials have become topics of great interest because of their unique properties and possible uses in technological applications. One powerful example is the embracement of "interfacial organic chemistry" by the materials science community.

Objective: This study was mainly directed towards incorporating solution phase reaction systems into the structure of self-assembled monolayers on gold nanoparticles to develop strategies to exploit the interfacial reactions of these systems to serve as probes to aid in our basic understanding of the mechanistic factors that control molecular interactions and organic reactions at organized monolayer interfaces.

Method: A series of aldehyde- and azide-terminated alkanethiols with varied alkyl chain lengths were synthesized and successfully incorporated onto the surface of small gold nanoparticles through the place-exchange reaction.

Results: Employing NMR technique, the physical organic chemistry aspects of these tailored gold nanoparticles, especially the interfacial reactivity of them for the imine formation and 1,3-dipolar cycloaddition reactions were evaluated in some detail. Furthermore, the effect of electronic parameter on these interfacial reactions was probed, and it was found that this parameter plays a key role and controls the progress of reaction. Synthesis and characterization of modified nanoparticles were studied by 1HNMR, TEM, FTIR, and UV/Vis technique.

Conclusion: In summary, this study has tried to extend the simple organic synthetic pathways into the surface chemistry of nanoparticles and also two well-known organic reactions; namely click reaction and imine formation on the surface of nanoparticles could be carried out under ambient conditions.
\end{abstract}

Keywords: Functionalized gold nanoparticles, interfacial organic reactions, iimine reaction, cycloaddition reaction, modification, $1 \mathrm{H}-\mathrm{NMR}$. 